\title{
Gambling and Personality Dimensions
}

\author{
Brian L. Odlaug • Samuel R. Chamberlain
}

Published online: 22 December 2013

(C) Springer International Publishing AG 2013

\begin{abstract}
Gambling dates back to ancient times, yet new arenas for gambling, such as the Internet, and methods of assessing psychiatric illness in the modern age have shifted our understanding of gambling as an addiction. Accordingly, Gambling Disorder is now a part of the Addictive Disorders in the DSM-5, which has further catalyzed a debate over the contribution of personality traits (rather than just personality disorders) to the manifestation and maintenance of psychiatric conditions such as Gambling Disorder. This selective review considers relationships between gambling and personality traits. The possible existence of distinct subtypes of Gambling Disorder, defined via personality traits, is highlighted, along with consideration of whether objective neurocognitive markers could serve as proxy markers of 'personality' more amenable to scientific dissection rather than relying on questionnaire-based measures alone. The clinical utility of subtyping and future areas of research are discussed.
\end{abstract}

Keywords Gambling disorder - Cognition · Compulsivity · Dimensions · Impulsivity $\cdot$ Personality $\cdot$ Self-regulation

\section{Introduction}

The most significant update to the field of gambling research over the past year occurred in May of 2013 when the $5^{\text {th }}$

\section{B. L. Odlaug $(\triangle)$}

Department of Public Health, Faculty of Health and Medical

Sciences, University of Copenhagen, Øster Farimagsgade 5A, 1014 Copenhagen K, Denmark

e-mail: brod@sund.ku.dk

\section{S. R. Chamberlain}

Department of Psychiatry \& MRC / Wellcome Trust Behavioural and Clinical Neurosciences Institute, University of Cambridge, Cambridge, UK

e-mail: srchamb@gmail.com

S. R. Chamberlain

Cambridge and Peterborough NHS Foundation Trust (CPFT),

Cambridge, UK edition of the Diagnostic and Statistical Manual of Mental Disorders (DSM-5) reclassified Gambling Disorder (GD) as a "Non-Substance-Related Addictive Disorder" [1••]. This nosological reclassification was in recognition of clinical and neurobiological similarities between substance use disorders (SUDs) and GD. Changes from the $4^{\text {th }}$ edition of the DSM to DSM-5 included dropping the 'illegal acts' criterion [1••]; a change which has been found to have no impact on the overall integrity of the GD diagnosis $[2 \cdot \bullet, 3]$.

The recognition in DSM-5 that GD may share neurobiological and pathophysiological characteristics similar to substance addictions may aid in developing efficacious treatments for GD. Currently, there are no FDA-approved medications for GD, although the most effective pharmacotherapy to date appears to be opioid antagonists, including naltrexone and nalmefene $[4 \cdot 5]$, a class of medications also indicated for the treatment of alcohol or opiate dependence. Our understanding of the impact of psychiatric conditions and personality components, such as impulsivity and novelty-seeking, on the development and maintenance of GD is, however, relatively limited at this time. Psychiatric conditions, such as depression, anxiety, and other addictions, are extremely common in GD and have been postulated as contributing to both the development and maintenance of GD over the lifespan [6]. By understanding the impact of these personality traits and potentially subtyping patients based upon both clinical and personality characteristics, treatments may be tailored to the individual needs of patients and subsequently increase the success of first-time treatment for GD. This paper seeks to selectively review the recent literature on gambling and personality as it relates to treatment considerations and research directions.

\section{Personality Subtypes: Are Certain Individuals Predisposed to Gamble?}

In the DSM-5, a greater emphasis was placed on personality traits and the impact of these traits on overall functioning, as opposed to assessing for either the presence or absence of a 
formal personality disorder [7]. This shift away from personality disorders and emphasis on the identification of personality dimensions was prompted, at least in part, by the recognition that dimensional aspects of personality, such as impulsivity or sensation-seeking, are just as important in the development and chronicity of psychiatric pathology as a full disorder of personality. Studies of individuals with GD, for example, have consistently shown high rates of impulsivity and novelty-seeking [8], yet studies examining the prevalence of personality disorders in GD patients have found a considerable range of rates (between 23-92\% of gambling disorder patients have been shown to have at least one personality disorder) $[9 \bullet \bullet, 10]$. With a focus on personality dimensions and trait conditions, rather than on disorders, one can hope to better understand the development of GD in adolescence and young adulthood while examining the maintenance of the disorder into the later years of life to target patients for more efficacious treatments [11••]. As such, studies have sought to examine the constructs of personality dimensions in samples of GD patients in relation to aspects of cognitive or trait impulsivity, as a means of better understanding the etiology and pathophysiology of GD.

\section{Cognitive Impulsivity vs Impulsive Personality}

\section{Impulsivity}

Gambling disorder is defined by impulsive, recurrent, and maladaptive gambling behavior, and was even classified as an 'Impulse Control Disorder' in the late DSM-IV [12]. Impulsivity as a trait component of gambling is supported by a number of studies of treatment-seeking or community-based gamblers [13-15], although impulsivity itself has been shown to be a multi-faceted construct within GD [16]. In fact, a study examining the question of trait versus state impulsivity in gambling found that trait - rather than state - impulsivity was associated with GD in a small sample of 37 GD patients [17].

Aspects of trait impulsivity have also been explored using functional neuroimaging. In a functional magnetic resonance imaging (fMRI) study, 43 healthy adults underwent testing using a simulated slot machine to examine the neural impact of wins, losses, and near-misses as it related to measures of trait impulsivity. Subjects scoring high in measures of trait impulsivity (the I-7 impulsiveness scale, [18]) started a new game much faster and had heightened activation in the ventral striatum and amygdala following a win, demonstrating that trait impulsivity was associated with a divergent coding of winning within areas of the brain associated with dopaminergic function and reward; a result remarkably akin to that seen in substance addiction [19•].

Our understanding of the association between impulsivity and the development and maintenance of GD, however, is further complicated by research involving self-reported impulsivity, gender, environmental factors (such as socioeconomic status [SES]), and age of onset of gambling problems. For example, in a longitudinal study of 628 students followed for 8 years (beginning in the $7^{\text {th }}$ grade), self-reported impulsivity was an indicator for subsequent GD onset for those also reporting a low SES but not for those reporting a high SES [20]. A study of 1004 low SES males noted that self-reported impulsivity as measured by the Eysenck Impulsiveness Scale [21] and depressive symptoms at age 14 were associated with subsequent problematic gambling at age 17 [22].

\section{Personality Subtypes}

Personality subtypes - akin to subtyping based upon clinical characteristics - have been extensively explored in GD; the most documented of which is the conceptual, yet hypothetical, Pathways Model by Blaszcynski and Nower (2002) [23], which suggested three subtypes of problem or pathological gambler: a) behaviorally conditioned; b) emotionally vulnerable; and c) the antisocial impulsivist [23]. Similar personality subtypes predisposing individuals to the development of gambling disorder were suggested in an assessment of 229 patients with GD where Ledgerwood and Petry (2010) [11 ••] presented the same three personality subtypes for GD as proposed by the Pathways Model: behaviorally conditioned gamblers, emotionally vulnerable gamblers, and antisocial impulsivity gamblers. In both cases, the least severe form of GD comprises the behaviorally conditioned gambler, who develops GD based upon repeated exposure to gambling stimuli. Inadequate coping skills and the need to regulate emotional instability predisposes the emotionally vulnerable gambler to GD, while the antisocial impulsivity gambler is characterized by greater rates of psychopathology, impulsivity, and the need to regulate effects $[11 \bullet \bullet, 23]$. A similar study assessing 154 young adult patients with GD also identified three clusters of GD patients with heterogeneous dispersion amongst the sample [24•]. The first cluster $(47.3 \%$ of the sample) was the 'High General Functioning' gamblers who had the lowest levels of GD severity and with generally healthy personality traits. The second subtype, called the 'Depressive Type' ( $40 \%$ of the sample) was characterized as emotionally vulnerable with difficulties in coping with negative emotions and moderate levels of impulsivity. The third and smallest cluster, (12.7\% of the sample), called the 'Disorganized Type', had the most severe form of GD and displayed high rates of impulsivity, novelty-seeking, and harm avoidance [24•]. Given difficulties in the treatment of GD patients, including high levels of drop-out, the authors note that subtyping based upon gambling severity and psychopathological factors such as high levels of sensation-seeking or impulsivity [25] may be a prudent means of successfully engaging and treating patients more effectively [24•]. 
Gambling as a means of coping with negative effects such as a depressive state, as described in the emotionally vulnerable subtype of gambler, is commonly reported in GD as prompting gambling behavior for many individuals. Indeed, research has explored the possible existence of an 'emotionally vulnerable' subtype of GD. High levels of self-criticism have been found to exist in GD [26], and a link between negative coping skills and GD has been similarly reported [27]. In individuals reporting frequent gambling, those subjects found to be high in emotional vulnerability (e.g., affective and anxiety disorders, alcohol and substance dependence) were more likely to develop GD [28]. Similarly, in a study of 581 college students, negative emotionality was predictive of gambling-related cognitive distortions [29]. Further, in a large twin study (10,253 subjects), mathematical modeling indicated that GD and generalized anxiety disorder were substantially related in terms of genetic factors, and that environmental triggers were likely to differ between these two conditions [30].

\section{The Age of the Internet: A New Dimension in Gambling?}

Given the rapid rise in Internet gambling over the past ten years, it is important to question whether certain personality subtypes are more prone to engagement in this type of gambling, which is traditionally solitary and isolated [31]. Does the 'typical' Internet gambler fit within the context of the 'emotionally vulnerable' gambler as described by numerous researchers [11••, 23, 24•]? Could subtyping these individuals lead to better, more targeted treatments?

Gambling, in general, is a popular recreational activity worldwide, with a substantial percentage of the global population having reported gambling at least once in their lifetime. While the estimated lifetime prevalence of GD worldwide has remained consistent between 0.4-4.2\% [32,33] with higher rates in psychiatric outpatients $(10.2 \%$ men and $3.1 \%$ females) [34•] and college students (10.2\%) [35], the number of individuals reporting non-traditional forms of gambling (such as via the Internet) continues to increase, even though they remain illegal in many countries [36]. A recent survey in Australia noted that over $64 \%$ of a sample of 15,006 adults reported having gambled at least once in their lifetime, and $8.1 \%$ of individuals reported online gambling [37•].

Internet gambling may constitute a more severe form of pathology, although research into this area is quite limited to date. A Finnish study of 3,451 adults who had reported gambling over the past year found that those meeting criteria for GD were significantly more likely to report using both casino and Internet gambling avenues compared to both problem and non-problem gamblers who engage in casinoonly gambling [38••]. This is consistent with previous research suggesting that the more types of gambling a person engages in, the worse the gambling severity (although importantly, this is not exclusive to Internet gambling) [39]. A second Finnish study, which randomly sampled 2,826 adults, found that Internet gambling alone was significantly associated with more severe gambling problems compared to other forms of gambling (e.g., casino gambling) $[40 \bullet \bullet]$, suggesting the need for more Internet gambling legislation [31].

The Internet appears to disproportionately affect adolescents and young adults, where problematic Internet use behaviors have been shown to coincide with problematic gambling behaviors [41]. What is less known, however, is whether a certain subtype of individual may be predisposed to engaging in Internet gambling and developing problematic gambling behaviors as a result. A study of 1,102 Swiss adolescents found that the risk of problem gambling was increased for those who were male, abusing alcohol, and reported problematic Internet use [41], emphasizing the importance for clinicians to screen young adults for signs and symptoms of these related addictive behaviors. Children and adolescents genetically or environmentally primed for the development of addictive behaviors - whether substance or behavioral - may find the Internet to be a convenient and readily available avenue to engage in an addictive behavior such as gambling; prior to the advent of this technology, it is likely that such individuals would not have developed an addictive habit until a number of years later. Research into the impact of genetics and personality dimensions on disordered gambling - including Internet gambling - in a large sample of twins found that differences in personality traits (in particular negative emotionality, aggression, and alienation) contributed to over $40 \%$ of the genetic risk for the development of problematic gambling, although the authors concluded that a large proportion of genetic variation cannot be explained by personality characteristics [42•]. Questions remain regarding whether certain personality subtypes exist in children and adolescents which may put them at particular risk for the development of problematic gambling behaviors and should constitute a public health research priority in the coming years.

\section{Limitations of Traditional Personality Trait Measures: Towards Translational Markers}

Traditional measures of personality traits, such as those relating to impulsivity and venturesomeness, have - as demonstrated above - proven very valuable in exploring predisposing, and perpetuating personality influences over GD. It can be difficult, however, to relate these personality traits to the neurobiological processes within the brain (i.e., particular neurochemical systems and neuroanatomical substrates); this is partially due to 
the fact that traditional concepts relating to personality are rather 'meta-cognitive' in nature, and partially because they often involve subjective questionnaires and rating scales that are difficult to adapt for use in conjunction with techniques from the neurosciences (notably neuroimaging and pharmacological manipulations). Additionally, it is very problematic to translate traditional personality measures into animal models (and back), since these processes in nonhumans are generally inaccessible.

For these reasons, it is important to consider whether objective cognitive tests might serve as proxy personality (i.e. 'trait') markers that exist prior to the development of pathology and render people pre-disposed [43, 44]. This research approach shifts away from questionnaire-based measures in favor of objective, computerized, cognitive paradigms whose neurobiological substrates have, in many cases, been studied across species. Research has noted that patients with GD often show impaired decision-making on objective cognitive tests [45], and dysregulation of neural circuitry implicated in decision-making is central to neurobiological models of GD [46, 47]. For a cognitive measure to represent a 'trait' marker, it should exist not only in affected patients (people with GD), but also prior to development of the pathological form; for the latter, one can study either clinically unaffected first-degree relatives of patients, or - more pragmatically - milder forms of gambling ("subsyndromal" GD).

To the authors' knowledge, no studies have been published to date that have examined cognitive functioning in unaffected first-degree relatives of patients with GD. Several studies have, however, quantified cognition in people with a subsyndromal form of GD, as defined by the basis of total scores on the Structured Clinical Interview for DSM-IV Pathological Gambling (SCI-PG), a 10-item scale which quantifies the number of DSM-IV criteria met for pathological gambling [48]. In a study conducted in non-treatment-seeking young adults aged 1829 years, individuals at-risk of developing GD (defined as having SCI-PG scores of 1-2) showed significant decisionmaking deficits on the Cambridge Gamble Test (CGT, $n=74$ ) compared to young adults meeting 0 criteria on the SCI-PG $(n=112)$ [49]. In particular, at-risk subjects gambled more points, were more likely to bankrupt, and made more irrational decisions under situations of risk ambiguity. Thus, decision-making deficits predated the development of GD, suggesting that dysfunction of orbitofronto-limbic cortical circuitry may exist in people at-risk of GD even before it develops. In a related study, young adults with GD $(n=46)$, atrisk gamblers $(n=69)$, and controls $(n=135)$ were compared in terms of performance on a stop-signal test of response inhibition, and set-shifting test of cognitive flexibility [50]. It was found that subjects with GD manifested profound impairments on these two cognitive abilities, while the at-risk gamblers remained completely intact on both cognitive paradigms.
These data, although provisional, suggest that decisionmaking task measures may be more suited as candidate vulnerability (trait) markers for gambling problems than tests tapping these other domains. This is important to note since impaired response inhibition and cognitive flexibility are trait markers for other conditions, notably obsessive-compulsive disorder (OCD) [44], suggesting that vulnerability markers may differ between OCD and GD; a debated member of an Obsessive-Compulsive "spectrum" of disorders.

While cognitive measures may prove useful as candidate vulnerability markers for psychiatric conditions such as GD, the relationship between cognition and personality is not well established. For example, while 'personality' aspects relating to impulsivity are typically quantified using questionnairebased rating scales (e.g. the Barratt Impulsivity Scale), cognitive tests tapping impulsivity (e.g. the stop-signal paradigm) are quite reductionist in nature. These two forms of impulsivity do appear to be dissociable, in that patients can show elevated impulsivity on one measure but not the other, and vice versa. Further research to clarify how personality traits relate to cognitive measures will be important, as it may be that a combination of these different types of vulnerability markers could yield greater power to select targeted treatments on an individual level.

\section{Conclusions: Can a Dimensional Approach to Personality Improve Treatment Outcomes?}

Although the literature reviewed here shows that certain personality characteristics are common in GD, including impulsivity in various forms, the question remains as to whether subtyping based on personality characteristics could improve our ability to triage patients for appropriate care based upon their personality profile. Research has shown that a large percentage of GD patients discontinue treatment [51], and while a limited amount of research has explored subsequent reasons for treatment drop-out, the literature does indicate that personality may indeed have an impact on treatment outcome. Within the realm of personality, several studies have indicated that higher levels of clinician-rated or self-reported impulsivity have an impact on treatment discontinuation. A sample of 112 gambling disorder patients undergoing cognitive behavioral therapy (CBT) for GD noted that high levels of impulsivity at the initiation of treatment were positively correlated with treatment discontinuation [52]. Similarly, in a sample of 88 adult patients with GD, deficits in self-regulation (including impulsivity) were significantly related to higher rates of dropping out from CBT [53]. Further, a study of 127 treatment-seeking gamblers found a positive correlation between personality traits of novelty-seeking and dropping out of treatment [25]. Tailoring treatments, or at a minimum incorporating therapeutic 
components targeting impulsive thoughts or behaviors, may be warranted in certain patients with GD.

Other aspects of cognition, however, including cognitive distortions (i.e., erroneous beliefs about gambling outcome, a common problem in GD [47]), have been shown to be positively correlated with gambling relapse [54], although the personality subtypes of individuals exhibiting such distortions are poorly understood at this time. Questions remain whether certain treatments (whether pharmacotherapeutic, psychosocial support, or a combination of treatment approaches) may be preferential for certain subtypes of individuals with GD. Future research addressing aspects of personality traits in GD enhanced by neurocognitive or other objective measures is required in order to tease out the core features of GD as they relate to the development, maintenance, and treatment of the disorder across the lifespan.

Acknowledgments This research was supported by a research grant from the Trichotillomania Learning Center to Mr. Odlaug. Mr. Odlaug has received a research grant from the Trichotillomania Learning Center, has consulted for Lundbeck Pharmaceuticals, and has received honoraria and royalties from Oxford University Press. Dr. Chamberlain has consulted for Cambridge Cognition, P1Vital, and Shire Pharmaceuticals; and has received speaker honoraria from Lilly.

\section{Compliance with Ethics Guidelines}

Conflict of Interest Dr. Odlaug was a consultant for Lundbeck Pharmaceuticals and received a grant from Trichotillomania Learning Center. Dr. Odlaug received an honoraria and royalties from Oxford University Press and served as a consultant for Cambridge.

Human and Animal Rights and Informed Consent This article does not contain any studies with human or animal subjects performed by any of the authors.

\section{References}

Papers of particular interest, published recently, have been highlighted as:

- Of importance

-• Of major importance

1.• American Psychiatric Association. Diagnostic and Statistical Manual of Mental Disorders, $5^{\text {th }}$ edition. Washington, D.C.: Author; 2013. Gambling disorder was re-classified as a "Non-Substance-Related Addictive Disorder" from an "Impulse Control Disorder" in the DSM-IV.

2.• Denis C, Fatséas M, Auriacombe M. Analyses related to the development of DSM-5 criteria for substance use related disorders: 3. An assessment of Pathological Gambling criteria. Drug Alcohol Depend. 2012;22:22-7. A study of 161 gamblers finding that dropping the "illegal acts" criteria from the diagnostic criteria for DSM-5 Gambling Disorder did not impact the integrity of the diagnosis.

3. Petry NM, Blanco C, Stinchfield R, Volberg R. An empirical evaluation of proposed changes for gambling diagnosis in the DSM-5. Addiction. 2013;108:575-81.
4. Hodgins DC, Stea JN, Grant JE. Gambling disorders. Lancet. 2011;378:1874-84. A review of the current status of gambling disorder with discussions of psychosocial and pharmacotherapeutic treatment considerations.

5. Grant JE, Odlaug BL, Schreiber LR. Pharmacological treatments in pathological gambling. Br J Clin Pharmacol 2012, Sep 14 [Epub ahead of print].

6. Aragay N, Roca A, Garcia B, et al. Pathological gambling in a psychiatric sample. Compr Psychiatry. 2012;53:9-14.

7. American Psychiatric Association. Highlights of changes from DSM-IV-TR to DSM-5. Washington, D.C.: American Psychiatric Publishing; 2013. Available at http://www.dsm5.org/Documents/ changes $\% 20$ from $\% 20 \mathrm{dsm}$-iv-tr $\% 20$ to $\% 20 \mathrm{dsm}-5$.pdf. Accessed August 2013.

8. Michalczuk R, Bowden-Jones H, Verdejo-Garcia A, Clark L. Impulsivity and cognitive distortions in pathological gamblers attending the UK National Problem Gambling Clinic: a preliminary report. Psychol Med. 2011;41:2625-35.

9.• Odlaug BL, Schreiber LR, Grant JE. Personality disorders and dimensions in pathological gambling. J Pers Disord. 2012;26: 381-92. Personality disorders were noted in $45.5 \%$ of GD patients, although the presence of a personality disorder was not correlated with overall gambling symptom severity.

10. Bagby RM, Vachon DD, Bulmans E, Quilty LC. Personality disorders and pathological gambling: a review and re-examination of prevalence rates. J Pers Disord. 2008;22:191-207.

11.• Ledgerwood DM, Petry NM. Subtyping pathological gamblers based on impulsivity, depression, and anxiety. Psychol Addict Behav. 2010;24:680-8. Based upon characteristics of patients, the authors developed three subtypes of gambling disorder patients, which could prove useful in the context of treatment considerations.

12. American Psychiatric Association. Diagnostic and Statistical Manual of Mental Disorders, $4^{\text {th }}$ edition. Washington, DC: Author; 2000.

13. Álvarez-Moya EM, Ochoa C, Jiménez-Murcia S, et al. Effect of executive functioning, decision-making and self-reported impulsivity on the treatment outcome of pathological gambling. J Psychiatry Neurosci. 2011;36:165-75.

14. Hwang JY, Shin YC, Lim SW, et al. Multidimensional comparison of personality characteristics of the big five model, impulsiveness, and affect in pathological gambling and obsessive-compulsive disorder. J Gambl Stud. 2012;28:351-62.

15. Walther B, Morgenstern M, Hanewinkel R. Co-occurrence of addictive behaviours: personality factors related to substance use, gambling and computer gaming. Eur Addict Res. 2012;18:167-74.

16. Grall-Bronnec M, Wainstein L, Feuillet F, et al. Clinical profiles as a function of level and type of impulsivity in a sample group of at-risk and pathological gamblers seeking treatment. J Gambl Stud. 2012;28:239-52.

17. Lai FD, Ip AK, Lee TM. Impulsivity and pathological gambling: is it a state or a trait problem. BMC Res Notes. 2011;13:492.

18. Eysenck SGB. The I7: development of a measure of impulsivity and its relationship to the superfactors of personality. In The impulsive client: theory, research and treatment. Edited by McCown WG, Johnson JL, Shure MB. Washington DC: American Psychological Association; 1993.

19. Shao R, Read J, Behrens TE, Rogers RD. Shifts in reinforcement signalling while playing slot-machines as a function of prior experience and impulsivity. Transl Psychiatry. 2013;3:e235. A recent fMRI study which noted that trait impulsivity was associated with a divergent coding of winning within areas of a the brain associated with dopaminergic function and reward; a result remarkably similar to that seen in substance addiction.

20. Auger N, Lo E, Cantinotti M, O’Loughlin J. Impulsivity and socioeconomic status interact to increase the risk of gambling onset among youth. Addiction. 2010;105:2176-83. 
21. Eysenck SB, Eysenck HJ. Impulsiveness and venturesomeness: their position in a dimensional system of personality description. Psychol Rep. 1978;43:1247-55.

22. Dussault F, Brendgen M, Vitaro F, et al. Longitudinal links between impulsivity, gambling problems and depressive symptoms: a transactional model from adolescence to early adulthood. J Child Psychol Psychiatry. 2011;52:130-8.

23. Blaszczynski A, Nower L. A pathways model of problem and pathological gambling. Addiction. 2002;97:487-99.

24. Jiménez-Murcia S, Granero R, Stinchfield R, et al. Typologies of young pathological gamblers based on sociodemographic and clinical characteristics. Compr Psychiatry. 2013;54:1153-60. A study assessing 154 young adult patients with GD also identified three clusters of GD patients similar to those noted by Ledgerwood \& Petry, with heterogeneous dispersion amongst the sample.

25. Smith D, Harvey P, Battersby M, et al. Treatment outcomes and predictors of drop out for problem gamblers in South Australia: a cohort study. Aust N Z J Psychiatry. 2010;44:911-20.

26. Pascual-Leone A, Gomes K, Orr ES, et al. Affective and cognitive correlates of gambling behavior in university students. J Gambl Stud. 2011;27:401-8.

27. Afifi TO, Cox BJ, Martens PJ, Sareen J, Enns MW. Demographic and social variables associated with problem gambling among men and women in Canada. Psychiatric Res. 2010;178:395-400.

28. Hodgins DC, Schopflocher CP, Martin CR, et al. Disordered gambling among higher-frequency gamblers: who is at risk? Psychol Med 2012:1-12. [Epub ahead of print].

29. King SM, Abrams K, Wilkinson T. Personality, gender, and family history in the prediction of college gambling. J Gambl Stud. 2010;26:347-59.

30. Giddens JL, Xian H, Scherrer JF, et al. Shared genetic contributions to anxiety disorders and pathological gambling in a male population. J Affect Disord. 2011;132:406-12.

31. Bonnaire C. Internet gambling: what are the risks? Encéphale. 2012;38:42-9.

32. Lorains FK, Cowlishaw S, Thomas SA. Prevalence of comorbid disorders in problem and pathological gambling: systematic review and meta-analysis of population surveys. Addiction. 2011;106:490-8.

33. Liu L, Luo T, Hao W. Gambling problems in young people: experience from the Asian region. Curr Opin Psychiatry. 2013;26:310-7.

34. Nehlin C, Grönbladh L, Fredriksson A, Jansson L. Alcohol and drug use, smoking, and gambling among psychiatric outpatients: a 1-year prevalence study. Subst Abus. 2013;34:162-8. A recent prevalence study of gambling disorder in psychiatric outpatients.

35. Nowak DE, Aloe AM. The prevalence of pathological gambling among college students: A meta-analytic synthesis, 2005-2013. J Gambl Stud 2013. [Epub ahead of print].

36. Goudriaan AE. Gambling and problem gambling in the Netherlands. Addiction 2013. Jun 6 [Epub ahead of print].

37. Gainsbury SM, Russell A, Hing N, et al. How the Internet is Changing Gambling: Findings from an Australian Prevalence Survey. J Gambl Stud 2013. A recent survey of over 15,000 adults in Australia noting high rates of any lifetime gambling (over $64 \%$ ) and Internet (8.1\%) gambling.

38.• Castrén S, Basnet S, Salonen AH, et al. Factors associated with disordered gambling in Finland. Subst Abuse Treat Prev Policy. 2013;8:24. A Finnish study of 3,451 adults who had reported gambling over the past year found that those meeting criteria for a GD were significantly more likely to report using both casino and Internet gambling avenues compared to both problem and nonproblem gamblers who engage in casino-only gambling.

39. Laplante DA, Nelson SE, Gray HM: Breadth and Depth Involvement: Understanding Internet Gambling Involvement and Its Relationship to Gambling Problems. Psychol Addict Behav 2013. [Epub ahead of print]

40.• Castrén S, Basnet S, Pankakoski M, et al. An analysis of problem gambling among the Finnish working-age population: a population survey. BMC Public Health. 2013;13:519. A Finnish study of 2,826 adults which found that Internet gambling alone was significantly associated with more severe gambling problems compared to other forms of gambling.

41. Tozzi L, Akre C, Fleury-Schubert A, Suris JC. Gambling among youths in Switzerland and its association with other addictive behaviours. A population-based study. Swiss Med Wkly. 2013;143: w13768.

42. Slutske WS, Cho SB, Piasecki TM, Martin NG. Genetic overlap between personality and risk for disordered gambling: evidence from a national community-based Australian twin study. J Abnorm Psychol. 2013;122:250-5. A large twin study which noted that personality traits (in particular negative emotionality, aggression, and alienation) contributed to over $40 \%$ of the genetic risk for the development of problematic gambling.

43. Gottesman II, Gould TD. The endophenotype concept in psychiatry: etymology and strategic intentions. Am J Psychiatry. 2003; $160: 636-45$.

44. Chamberlain SR, Menzies L. Endophenotypes of obsessivecompulsive disorder: rationale, evidence and future potential. Expert Rev Neurother. 2009;9:1133-46.

45. van Holst RJ, van den Brink W, Veltman DJ, Goudriaan AE. Why gamblers fail to win: a review of cognitive and neuroimaging findings in pathological gambling. Neurosci Biobehav Rev. 2010;34:87-107.

46. Grant JE, Brewer JA, Potenza MN. The neurobiology of substance and behavioral addictions. CNS Spectr. 2006;11:924-30.

47. Clark L. Decision-making during gambling: an integration of cognitive and psychobiological approaches. Philos Trans R Soc Lond B Biol Sci. 2010;365:319-30.

48. Grant JE, Steinberg MA, Kim SW, Rounsaville BJ, Potenza MN. Preliminary validity and reliability testing of a structured clinical interview for pathological gambling. Psychiatry Res. 2004;128:79-88.

49. Grant JE, Chamberlain SR, Schreiber LR, et al. Selective decisionmaking deficits in at-risk gamblers. Psychiatry Res. 2011;189:115-20.

50. Odlaug BL, Chamberlain SR, Kim SW, et al. A neurocognitive comparison of cognitive flexibility and response inhibition in gamblers with varying degrees of clinical severity. Psychol Med. 2011;41:2111-9.

51. Grant JE, Kim SW, Kuskowski M. Retrospective review of treatment retention in pathological gambling. Compr Psychiatry. 2004;45:83-7.

52. Leblond J, Ladouceur R, Blaszczynski A. Which pathological gamblers will complete treatment? Br J Clin Psychol. 2003;42: 205-9.

53. Álvarez-Moya EM, Jiménez-Murcia S, Aymamí MN, et al. Subtyping study of a pathological gamblers sample. Can J Psychiatry. 2010;55: 498-506.

54. Ladouceur R, Sylvain C, Boutin C, et al. Cognitive treatment of pathological gambling. J Nerv Ment Dis. 2001;189:774-80. 